\title{
Karyotype and Pattern of Chromosome Replication in Lagurus lagurus Pall (Cricetidae, Microtinae)
}

\author{
M. R. Baneriee ${ }^{1}$ and Ruby J. Walker \\ Biology Branch, Carcinogenesis, Etiology, National Cancer Institute, \\ Bethesda, Maryland 20014, U.S.A.
}

Received February 20, 1967

The potential value of chromosome characters in mammalian taxonomy has been limited by the inadequate knowledge of the chromosomes of various species listed by the systematists (Makino 1951). In recent years some cytologists have become interested in studying the karyotype of different mammalian species (Klinger 1963, Nadler 1965). In addition, the need for detailed knowledge about the karyotype of mammals, particularly those species which are being investigated for possible experimental use, also has been emphasized by several authors (Haddow 1960, Mitchell 1960).

The above considerations led us to study the mitotic and meiotic chromosomes of an East European mammal, Lagurus lagurus Pall (Rodentia, Cricetidae, Microtinae). Since the introduction of $L$. lagurus as a test animal for chemical carcinogenesis these animals are being increasingly used in cancer research (Pogosianz et al. 1960, Weisburger et al. 1965). Diploid chromosome number of L. lagurus was first listed by Matthey (1954) and this was followed by a short report on the karyotype studied from embryonic cells grown in vitro (Pogosianz and Zaharov 1962). Improved methods of chromosome preparation now permit a more detail study of the chromosomes using primary tissues obtained directly from the adult animal. This report presents the results of our study on the structure and behavior of both mitotic and meiotic chromosomes as well as the pattern of DNA replica. tion in Lagurus lagurus (Steppe-Lemming).

\section{Materials and methods}

Adult Steppe-Lemmings (Lagurus lagurus Pall), 3 males and 3 females, were kindly made available to us by Dr. R. Yamamoto of the National Cancer Institute, Bethesda, Maryland, and by Dr. R. Whitney of Elmira College, Elmira, New York. These animals were the progeny of a stock originally obtained from the Institute of Experimental and Clinical Oncology, Academy of Medical Sciences, Moscow, U.S.S.R.

Chromosome preparation. Animals were injected with $0.2 \mathrm{ml}$ of $0.5 \%$ colchicine 1 hour before sacrifice. Spleen, testes and ovaries were used. Spleen and ovaries were minced into small pieces and suspended in distilled water for 10 minutes after which the tissues were fixed in $50 \%$ acetic acid, squashed and stained in acetic-orcein. Spermatogonial mitosis and male meiosis were studied in adult testis. Seminiferous tubules were freed from the outer tunica of the testis and part of the tubules were handled as described above. The remaining portion was prepared according to the air drying method of Evans et al., 1964.

Autoradiographs. Animals used for studying DNA synthesis, were administered $50 \mu \mathrm{c}$ $\left(6.7 \mathrm{C} / \mathrm{mM}\right.$, New England Nuclear Corp.) of thymidine ${ }^{3} \mathrm{H}$ by intraperitoneal injection, 3 hours later a second injection of $0.2 \mathrm{ml}$ of $0.5 \%$ colchicine was given also by intraperitoneal route. The same set of tissues was fixed in acetic alcohol $(1: 3)$, squashed and stained in

1 Present address: Department of Zoology and its Institute for Cellular Research, University of Nebraska, Lincoln, Nebraska, 68503, U.S.A. 
acetic orcein. Hypotonic pretreatment was as described before. Autoradiographs were prepared using Kodak ARIO stripping film and the slides were processed after 3 weeks of exposure.

\section{Results}

The diploid chromosome number of L. lagurus was determined as 54 with XX female and XY male sex-determining mechanisms (Table 1, Figs. 1 and 2). According to the position of the centromere and size of the chromosomes the 26 pairs of autosomes were classified as 1 pair of large submetacentric, 1 pair medium sub-metacentric, 2 pairs of small metacentric and 22 pairs of acrocentric chromosomes regularly decreasing in size (Figs. 3a and $3 \mathrm{~b})$. Almost all the acrocentric elements contained a short second arm and this was more prominent in the larger chromosomes of this group.
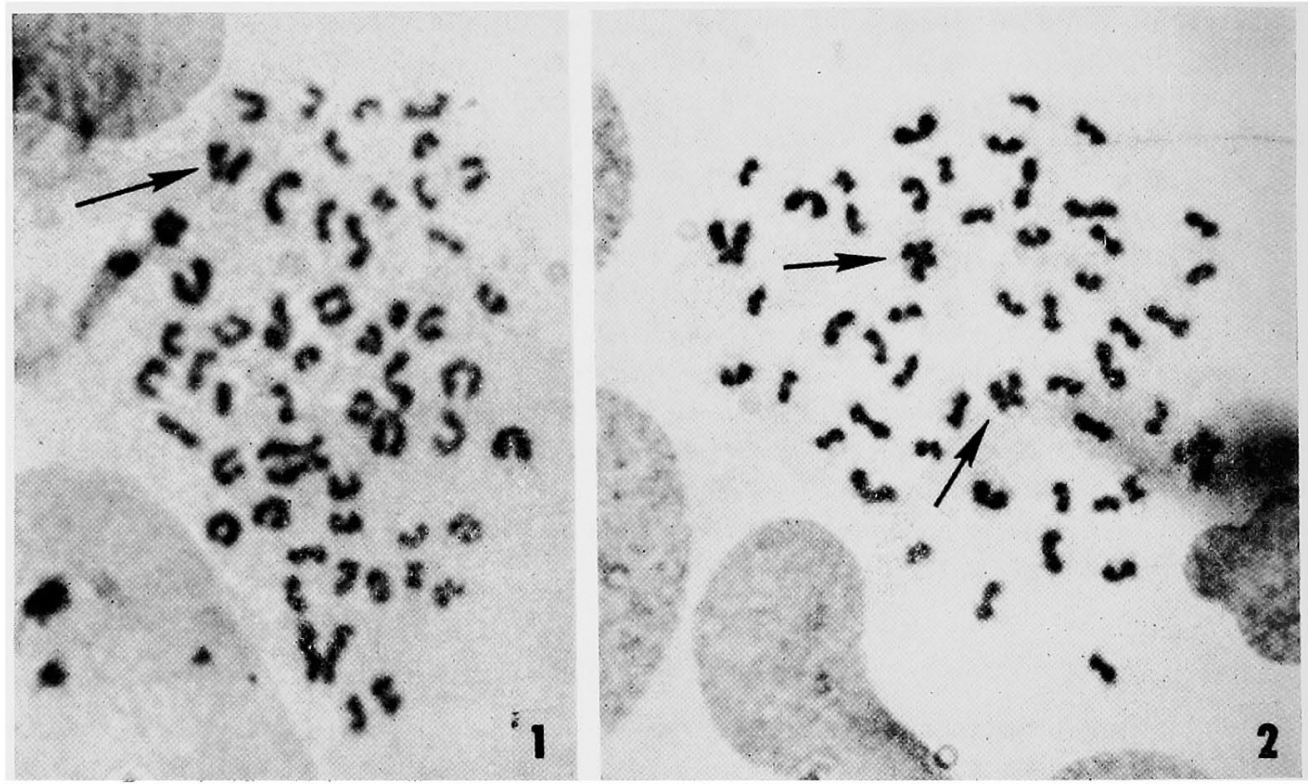

Figs. 1-2. 1, spermatogonial metaphase plate with 54 chromosomes. Arrow indicates the $\mathrm{X}$ chromosome (Squash). 2, metaphase plate from the ovary with 54 chromosomes. The $\mathrm{X}$-chromosomes are indicated by the arrows (Squash).

In addition to the medium size sub-metacentric chromosomes referred to above, male karyotype contained another medium sized, unpaired sub-metacentric element and an unpaired small acrocentric chromosome. In the female karyotype the same sub-metacentric chromosome was paired while no unpaired small acrocentric element was present. This medium sized sub-metacentric element, unpaired in the males and paired in the female, constitutes the $\mathrm{X}$ chromosome and the $\mathrm{Y}$ chromosome is contained in the group of small acrocentric elements. In the adult testes, majority of the spermatogonial metaphases contained 54 chromosomes (Table 1). However, in specimens 2 and 3 a small number of tetraploid and polyploid spermatogonial metaphases were also observed and Table 1 does not include these cells. During leptotene, zygotene and pachytene stages of meiotic prophase, the sex elements remained within 
a vesicle, usually located near the periphery of the nucleus (Fig. 4). At diakinesis, 26 autosomal bivalents along with the 2 sex chromosomes oriented

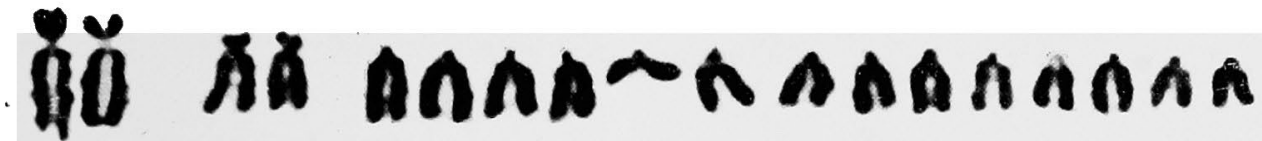

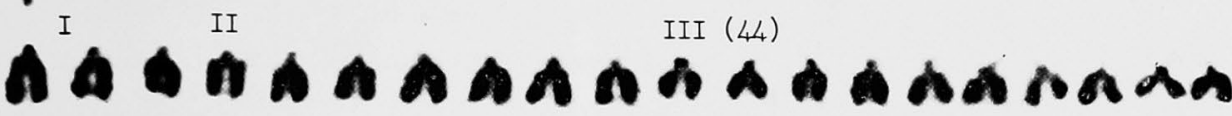

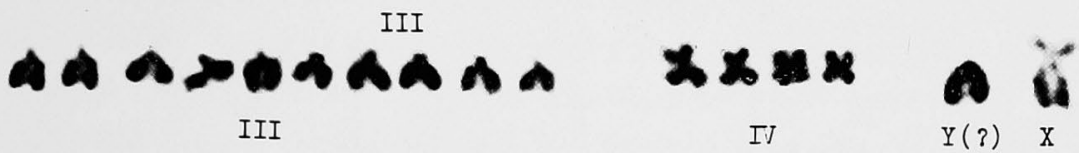

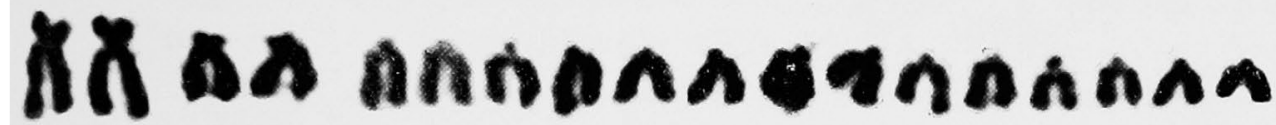
it

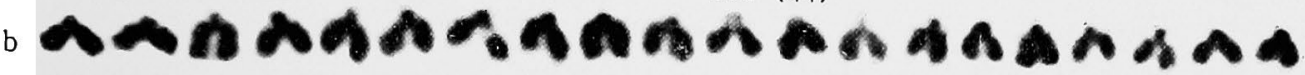

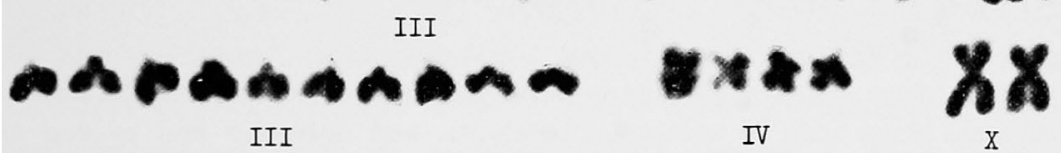

Fig. 3. Serial alignment of the metaphase chromosomes obtained from spleen. a, male. b, female.

Table 1. Diploid chromosome number in somatic and germ cells of Lagurus lagurus

\begin{tabular}{|c|c|c|c|c|c|c|c|c|c|c|}
\hline \multirow{2}{*}{ Sex } & \multirow{2}{*}{ Tissues } & \multirow{2}{*}{$\begin{array}{l}\text { Animal } \\
\text { number }\end{array}$} & \multirow{2}{*}{$\begin{array}{c}\text { Total } \\
\text { number } \\
\text { of cells } \\
\text { analyzed }\end{array}$} & \multicolumn{6}{|c|}{ Chromosome number } & \multirow{2}{*}{$\begin{array}{c}.0 \text { of cells } \\
\text { with } 54 \\
\text { chromosomes }\end{array}$} \\
\hline & & & & 51 & 52 & 53 & 54 & 55 & 56 & \\
\hline \multirow{6}{*}{ Male } & \multirow{3}{*}{ Spleen } & 1 & 31 & 1 & & 3 & 26 & 1 & & 84 \\
\hline & & 2 & 30 & & 4 & & 25 & & 1 & 81 \\
\hline & & 3 & 30 & 1 & & 2 & 27 & & & 90 \\
\hline & \multirow{3}{*}{ Testes } & 1 & 28 & 1 & & & 27 & & & 96 \\
\hline & & 2 & 29 & 1 & 1 & 1 & 26 & & & 89 \\
\hline & & 3 & 30 & 1 & 2 & & 27 & & & 90 \\
\hline \multirow{5}{*}{ Female } & \multirow{3}{*}{ Spleen } & 4 & 50 & 1 & & 1 & 47 & 1 & & 90 \\
\hline & & 5 & 30 & & 2 & 1 & 26 & & 1 & 84 \\
\hline & & 6 & 30 & 1 & 1 & 1 & 26 & 1 & & 84 \\
\hline & \multirow{2}{*}{ Ovary } & 5 & 22 & & 1 & & 20 & & 1 & 91 \\
\hline & & 6 & 26 & & & 1 & 25 & & & 96 \\
\hline
\end{tabular}

in an end to end association were clearly visible (Fig. 5). Autosomal bivalents usually contained at least one interstitial chiasma and at metaphase of first 


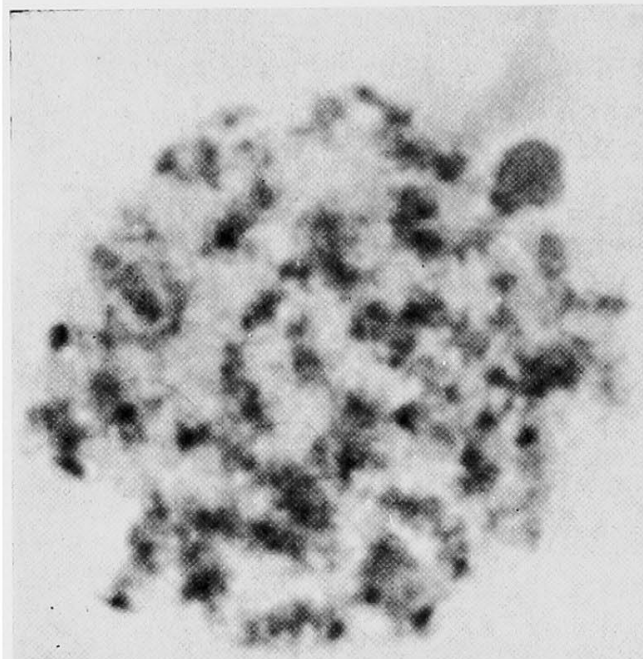

4

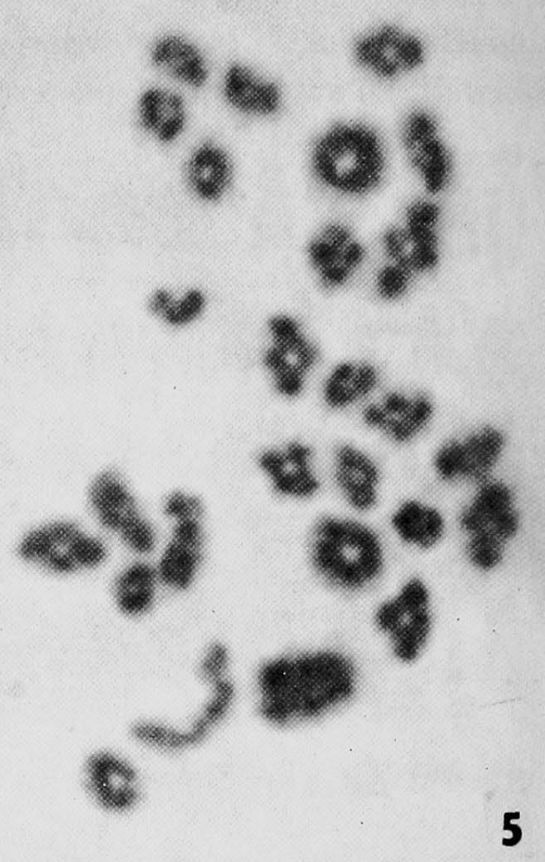

Figs. 4-5. 4, zygotene stage showing the sex-vesicle lying in the periphery of the nucleus (Squash). 5, diakinesis stage with 26 autosomal bivalents, and note the end to end association of the XY chromosomes (Squash).

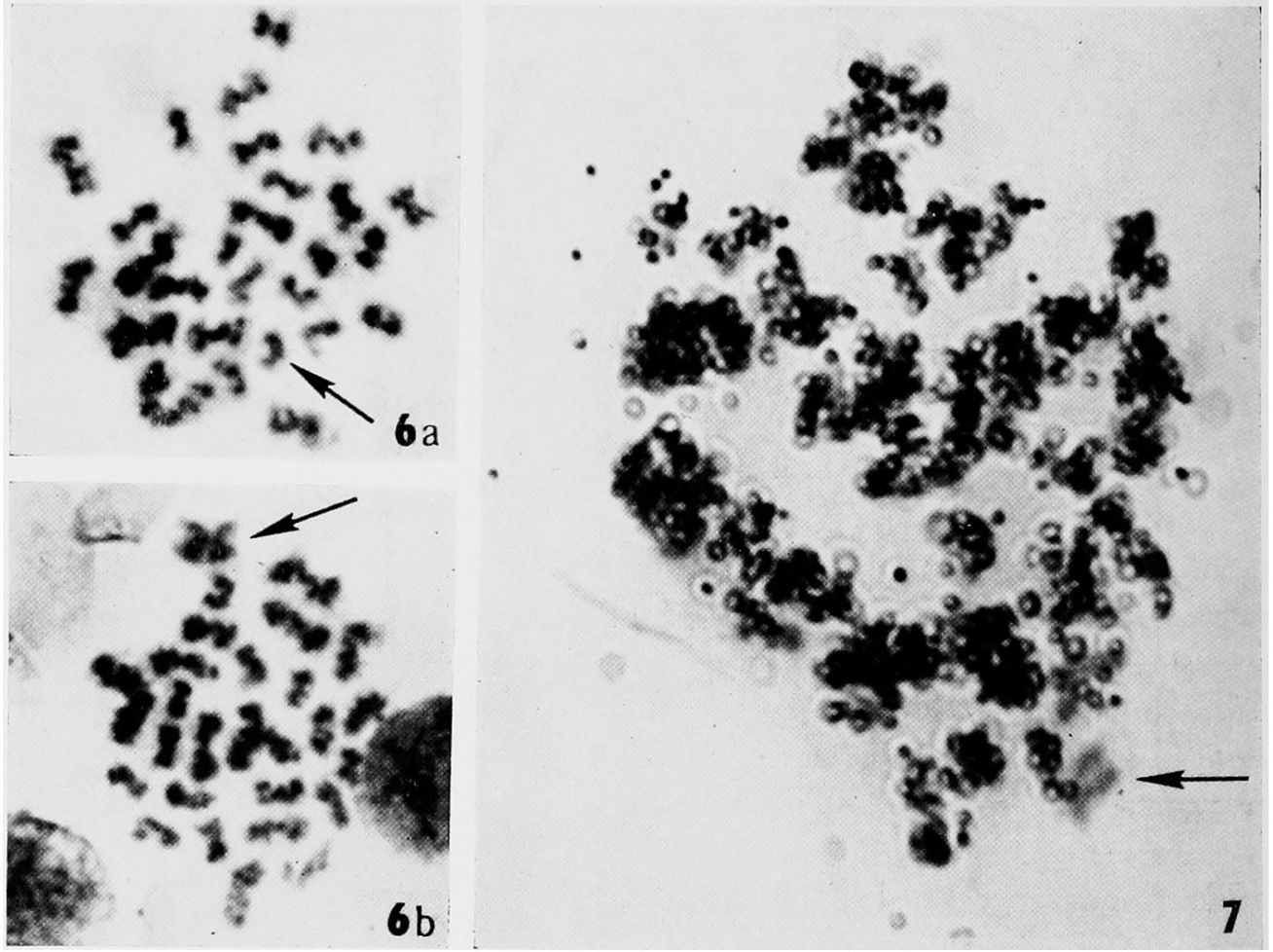

Figs. 6-7. 6, metaphase II Two cells each with 27 chromosomes. a, arrow indicates Y; $\mathrm{b}$, arrow indicates $\mathrm{X}$. 7, showing the asynchronous pattern of DNA replication. Autoradiograph of metaphase chromosomes obtained from spleen of a female. Note that one of the X-chromosomes is essentially unlabeled (arrow). 
spermatocyte division, most of the chiasmata in the smaller bivalents were in terminal position. Metaphase II contained 27 chromosomes and these are 26 autosomes and the $\mathrm{X}$ or $\mathrm{Y}$ chromosomes (Figs. 6a and 6b). The presence of two types of metaphase II cells thus indicates that in this species the sexchromosomes segregate reductionally at anaphase I. Although a small number of tetraploid and polyploid spermatogonial metaphases were observed in specimens 2 and 3 , no meiotic metaphase in these animals was found to contain an abnormal haploid chromosome number. Absence of any information on meiosis in L. lagurus led us to study the male meiotic chromosomes and the data did not reveal any features characteristic to this species. The prereductional segretation of the $\mathrm{X}$ and $\mathrm{Y}$ chromosomes and the end to end pairing of the same chromosomes during meiosis is in agreement with similar findings in other species of Microtinae (Matthey 1950, 1951).

Approximately 200 metaphases were examined from the autoradiographs of each of the 3 types of tissues and nearly $80-85 \%$ of the mitotic metaphases in the somatic tissues were labeled 4 hours after administration of the labeled DNA precursor, thymidine $-{ }^{3} \mathrm{H}$. However, at that time none of the spermatocyte prophase and metaphase cells in the testis were labeled. This may be because the 4 hour pulse after thymidine. ${ }^{3} \mathrm{H}$ treatment was too short for these cells to complete DNA synthesis, pass through $G_{2}$ phase of the cell cycle and reach mitosis. Time for DNA replication and $G_{2}$ phase in spermatocyte cells of some rodents was shown to be almost double that of the somatic cells (Utakoji and Hsu 1965). An asynchronous labeling pattern was observed among the metaphase chromosomes and also within the different regions of a single chromosome of the somatic cells (Fig. 7) and this observation is consistent with similar findings in other rodent species (Hsu et al. 1964).

\section{Discussion}

The present study revealed that the diploid chromosome number of Lagurus lagurus is 54 with $\mathrm{XY}$ male and XX female sex-determining mechanism and these results confirm the findings of the earlier investigators (Matthey 1957, Pogosianz and Zaharov 1962). The karyotype analysis of the metaphase chromosomes of L. lagurus is in general agreement with Pogosianz and Zaharov (1962). However, these authors classified one pair of acrocentric elements as distinctly larger than the rest of the chromosomes of the same group. During the present study it was observed that although the size of the acrocentric elements range from medium to small, the difference between the individual pairs of acrocentric chromosomes is very subtle and gradual, and it was not possible to distinguish a larger pair. All the acrocentric elements thus were arranged in the order of their decreasing size (Figs. 3a and $3 \mathrm{~b})$. The characteristic size and morphology of the $\mathrm{X}$ chromosomes permit a positive identification of those elements both in the mitotic and 
meiotic metaphases (Figs. 3a and 3b,6a and 6b). However, the Y-chromosome, being a member of the group of small acrocentric elements, cannot be identified positively in the somatic cells and the chromosome shown in the serial alignment of the male karyotype is entirely arbitrary (Fig. 3a).

Matthey (1957) reported that the "fundamental number" (N.F.) of chromosomes in L. lagurus is $56-58$, but the present data show that the total number of chromosome arms (N.F.) in this species is at least 63 and 64 in males and females, respectively. It is generally accepted that animals with a higher number of chromosomes have more acrocentric element (White 1954), and centric fusion of Robertsonian type (Robertson 1916) plays an important role in the evolution of different chromosome number and form. Matthey (1957) in discussing the cytologic affinity of L. lagurus considered that the karyotype of this genus is primitive and basically similar to the genus Phenacomys $(2 \mathrm{n}=56, \mathrm{XY})$ of Microtinae except that a single centric fusion in L. lagurus has lowered the chromosome number from 56 to 54 . This hypothesis is based on the observation that N.F. in L. lagurus is 5658. However, the present data show that the N.F. in L. lagurus is 63 or 64 , thus lowering of the chromosome number of L. lagurus to $2 \mathrm{n}=54$ could have involved more than one centric fusion. This report presents the data on the karyotype of one species of the genus Lagurus and we feel that until information on the chromosomes of the other two species of this genus is available, further discussion on the evolution of chromosome number and form in the genus Lagurus would be rather premature at this point.

With regard to the use of $L$. lagurus as a laboratory animal, it may be pointed out that except for the positive identification of the X-chromosomes, the karyotype of Steppe-Lemming with a high chromosome number does not seem to provide any additional cytogenetic characteristics which might be advantageous over other rodents conventionally used in cancer research. The general pattern of chromosome replication also seems to be in agreement with other rodent species so far studied.

\section{Summary}

Karyotype from somatic and germ cells of Lagurus lagurus Pall (SteppeLemming) a species of Microtinae, showed 54 chromosomes in the diploid set with $X Y$ and $X X$ sex-chromosome mechanisms. The autosomes were classified into 3 morphological groups. The $\mathrm{Y}$ chromosome is a small acrocentric element and its identification in the male karyotype is rather difficult among the autosomes. Characteristic size and morphology of the X-chromosome, however, permits its positive identification. All the autosomal bivalents showed at diakinesis at least one chiasma and the $\mathrm{X}$ and the $\mathrm{Y}$ chromosomes are associated end to end. There are two types of metaphase II cells, a $Y$ class and an $\mathrm{X}$ class, resulting from the reductional segregation of the sex-chromosomes. The general pattern of DNA replication, both in somatic and germ 
cells is in agreement with other rodents so far studied.

Acknowledgment: The authors wish to express their sincere thanks to Dr. Hans L. Falk for his support during this investigation.

\section{References}

Haddaw, A. 1960. Fundamental programes in causation, prevention and therapy. Lab. Animal Centre (England) Coll. Pap. 9: 11-23.

Hsu, T. C., Schmid, W. and Stubblefield, E. 1964. DNA replication sequence in higher animals. (The role of chromosomes in development, M. Locke, Editor, Academic Press, New York).

Klinger, H. P. 1963. The somatic chromosomes of some Primates. Cytogenetics 2: 140-151.

Makino, S. 1951. An atlas of the chromosome number in animals. Iowa State College Press, Ames, Iowa.

Matthey, R. 1950. Le chromosomes sexuels geants de Microtus agrestis. Cellule 53: 163-184.

- 1951. Chromosomes of Muridae. Experientia 7: 340-341.

- 1956. Cytologie comparee des Muridae. L'ongine des Ellobii. Experientia 12: 337-338.

- 1957. Analyse cytotaxonomique de huit expecas de Muridae. Arch. Klaus-Stift. VererbForsch 32: 385-404.

Mitchell, S. S. 1960. Introductory remarks. Lab. Animal Centre (England) Coll. Pap. 9: $1-10$.

Nadler, C. F. 1965. Chromosomes of the ground squirrels. Spermophilus boldingi and report of an aberrant karyotype in a phenotypically normal female. Cytogenetics 4: $37-44$.

Pogosianz, H. E., Bolonina, N. I. and Olshevskaja, L. V. 1960. The Steppe-Lemming (Lagurus lagurus Pall). A new animals for cancer research. Acta Un. Int. Cancer 16: 1238.

- and Zaharov. A. F. 1962. On the karyotype of the Steppe-Lemming. Zschr versuchstierk 1: $93-98$.

Robertson, W. R. B. 1916. Chromosome studies. I. Taxonomic relationships shown in the chromosomes Tettigonidae and Acrididae: V-shaped chromosomes and their significance in Acrididae, Locustidae and Cryllidae; chromosomes and variations. J. Morph. 27: 179-331.

Utakoji, T. and Hsu, T. C. 1965. DNA replication in somatic and germline cells of the male Chinese hamsters. Cytogenetics 4: 295-315.

Weisburger, J. H., Grantham, P. H. and Weisburger, E. K. 1965. Metabolism of 2-acetoaminofluorene in the Steppe-Lemming. British J. Cancer 19: 581-588.

White, M. J. D. 1954. Animal cytology and evolution. Cambridge University Press. 